\title{
Patent ductus arteriosus and effects of low oxygen saturation limits
}

\section{Journal of Perinatology (2009) 29, 529-530; doi:10.1038/p.2009.63}

The study of Noori $e t$ al. ${ }^{1}$ in this issue, brings together two of the major unresolved therapeutic issues in preterm neonatology, how much oxygen to give them and how to treat the patent ductus arteriosus. Oxygen saturations in the low 90s were accepted by most of us as appropriate for preterm babies until the publication of the observational study of Tin et al. ${ }^{2}$ in 2001 from the north of England. This study used different neonatal intensive care unit (NICU) policies to show a markedly lower rate of retinopathy of prematurity needing cryotherapy between babies with saturations targeted between 70 and $90 \%$ and those targeted between 88 and $98 \%$ (6 vs $27 \%$ ) with no difference in mortality. The study of Noori et $a .^{1}$ adds further consistency to the reductions in treated retinopathy of prematurity rates that have now been shown in several 'before and after' studies around a change in an NICU saturation targeting protocol. ${ }^{3,4}$ Noori et al.'s study ${ }^{1}$ shows a reduction of treated retinopathy of prematurity from 34 to $9 \%$, 4 years either side of a change in oxygen saturation limits from $89-94 \%$ to $83-89 \%$ in two NICUs in Los Angeles. The observational data with respect to the premature eye are becoming quite compelling but... (and there's always a 'but'), what is good for one organ may not be good for other organs. Two of the organs whose normal function may be very dependent on oxygen levels are the brain and the closure of the ductus arteriosus.

Noori et al.'s study ${ }^{1}$ explores the latter of these by comparing the incidence of what they term as haemodynamically significant patent ductus arteriosus (PDA) in the two epochs either side of the change in oxygen policy. They show a statistically significant but probably not clinically significant increase in incidence from 63 to $75 \%$ with no significant difference in ligation rate (24 vs 30\%). PDA is a difficult outcome because it depends how you look for it and, if you look echocardiographically, how you measure significance. The protocol in this study involved echocardiography within the first $24 \mathrm{~h}$, with a $1.0 \mathrm{~mm}$ diameter being used as a threshold for haemodynamic significance and treatment. This is quite a low threshold (a $1.0 \mathrm{~mm}$ duct is quite well constricted) and this reflects in a high incidence and treatment rate. Although there is no consensus, diameters between 1.5 and $2.0 \mathrm{~mm}$ are more commonly used in very preterm babies as cut offs for haemodynamic significance. In our own work, pulmonary to systemic flow ratios did not rise until ducts were over $1.5 \mathrm{~mm}$ in diameter and consistently so when over $2 \mathrm{~mm} .^{5}$ Notwithstanding this, the same criteria were applied in each time period in the Noori's study ${ }^{1}$ and there was a small rise in the incidence. There was also an increase in ligation rate but this did not reach statistical significance. However, to my non-North American eyes, the surgical ligation rates in both time periods are very high. To compare with my local data, in 2003 to 2006 inclusive, the median rate of PDA ligation in babies $<1000 \mathrm{~g}$ in the eight perinatal NICUs in New South Wales, Australia was $7.3 \%$ with a range from 4.6 to $15.9 \%$ (Source: NSW NICUS data collection). Either we, 'Down Under', are missing something or there are a lot of babies on the other side of the Pacific having unnecessary chest surgery. Like many aspects of PDA therapy, this badly needs testing in a clinical trial, particularly in light of the associations described from the trial of indomethacin prophylaxis in preterms (TIPP) trial between ductal ligation and worse pulmonary, retinal and 18-month neurosensory outcomes. ${ }^{6}$

The targeted early approach to medical PDA therapy used in this study has much theoretical merit but is also untested. We are testing this in the DETECT trial, which is currently recruiting in three Australian centers. In this trial, babies born before 29 weeks have echocardiography within the first $12 \mathrm{~h}$ and those with poorly constricting ducts (thresholds 1.2 to $2.0 \mathrm{~mm}$ depending on postnatal age) are blindly randomised to indomethacin or placebo. Hopefully, the results of this study will give better guidance to the value of an early targeted approach.

Despite these therapeutic uncertainties about the ductus, Noori et al. ${ }^{1}$ showed only small increases in PDA as defined in this study with lower oxygen saturation targeting. Although this is reassuring, the trend is there and, with greater statistical power, this might become more significant. The other remaining uncertainty about lower oxygen targeting is the impact on the developing brain. The original observational study of Tin $e t a l{ }^{2}$ is, to my knowledge, the only one that has looked at neurodevelopmental outcome. This study showed no differences in cerebral palsy rates at 1 year in babies whose oxygen saturations were targeted lower. This is an important observation but not an outcome or study design with which to confirm safety, so this uncertainty about the impact of lower oxygen targeting on the brain remains. To go to that well-worn clinical epidemiological conclusion... more research is needed. However, with this I do not have to leave you in the usual evidence-based vacuum because this research is happening. In an unprecedented international collaboration, several large and similarly designed trials are underway: BOOST 2 (Australia), BOOST UK and BOOST NZ, the Canadian Oxygen Trial and US 
SUPPORT Trial. The quickest way to get a definitive answer to the perennial question of how much oxygen to give these most vulnerable babies is to join one of these trials.

\section{Conflict of interest}

The author declares no conflicts of interest.

N Evans

Department of Newborn Care, Royal Prince Alfred Hospital and University of Sydney, Sydney, NSW, Australia

E-mail: nevans@med.usyd.edu.au

\section{References}

1 Noori S, Patel D, Friedlich P, Siassi B, Seri I, Ramanathan R. Effects of low oxygen saturation limits on the ductus arteriosus in extremely low birth weight infants. J Perinatol 2009; (in press).

2 Tin W, Milligan DWA, Pennefather P, Hey E. Pulse oximetry, severe retinopathy and outcome at one year in babies of less than 28 weeks gestation. Arch Dis Child 2001; 84: F106-F110.

3 Chow LC, Wright KW, Sola A, CSMC Oxygen Administration Study Group. Can changes in clinical practice decrease the incidence of severe retinopathy of prematurity in very low birth weight infants? Pediatrics 2003; 111: 339-345.

4 Vanderveen DK, Mansfield TA, Eichenwald EC. Lower oxygen saturation alarm limits decrease the severity of retinopathy of prematurity. J AAPOS 2006; 10: 445-448.

5 Evans NJ, Iyer P. Assessment of ductus arteriosus shunting in preterm infants requiring ventilation: Effect of inter-atrial shunting. J Pediatr 1994; 125: 778-785.

6 Kabra NS, Schmidt B, Roberts RS, Doyle LW, Papile L, Fanaroff A. Trial of indomethacin prophylaxis in preterms investigators. Neurosensory impairment after surgical closure of patent ductus arteriosus in extremely low birth weight infants: results from the trial of indomethacin prophylaxis in preterms. J Pediatr 2007; 150: 229-234.

(c) SOMERIIEHISRESERVED Alike 3.0 License. To view a copy of this license, visit http://creativecommons.org/licenses/by-nc-sa/3.0/ 